\title{
Fully Fabric Knitted Antennas for Wearable Electronics
}

\author{
Shiyu Zhang ${ }^{(1)}$, Alford Chauraya ${ }^{(1)}$, Rob Seager ${ }^{(1)}$, Yiannis C. Vardaxoglou* ${ }^{(1)}$, \\ William Whittow ${ }^{(2)}$, Tessa Acti ${ }^{(3)}$, Tilak Dias ${ }^{(3)}$ \\ (1) School of Electronic, Electrical and Systems Engineering, Loughborough University, \\ Loughborough, UK. Email: r.d.seager@lboro.ac.uk \\ ${ }^{(2)}$ Antrum Ltd, Rutland Hall, Enterprise Office, Loughborough University Campus, \\ LE11 3TU, UK. Email: will.whittow@antrum.co.uk \\ ${ }^{(3)}$ Centre for Research in Advanced Textiles (CReATe), School of Art and Design, \\ Nottingham Trent University, Nottingham, NG1 4BU, UK
}

The worldwide wearable technology market is expected to exceed $\$ 6 \mathrm{~B}$ by 2016 and wearable antennas will be used by the emergency services, fashion designers, military, athletes and patients. Exploring different methods of fabricating antennas is important especially as user comfort and aesthetics are key considerations in ensuring mainstream acceptance. Previously the authors have examined embroidered antennas using highly conductive threads [Chauraya et al. EuCAP 2012]. Please see this paper for a detailed literature review of wearable antennas.

In this paper, we examine knitting as a technique of fabricating antennas. These antennas were fully fabric with a knitted ground plane, a knitted substrate and a knitted patch element. They were fabricated using industrial knitting machinery and hence could potentially be scaled up to mass-manufacture. Four different versions were considered (all had a knitted ground plane and substrate): i) a conducting coated nylon fabric (Nora Dell); ii) a knitted patch with a high fiber density (Sample 1); iii) a knitted patch with a medium fiber density (Sample 2) and iv) a knitted patch with a coarse fiber density (Sample 3). The resulting antennas were extremely flexible and soft to the touch.

The return loss results are shown in the figure. All the antennas were fed with a probe feed positioned the same distance from the edge of the patch - the magnitude of the return loss could be improved by finding the optimal feeding point. The S11 and associated bandwidth results of the antennas suggests that the Nora Dell antenna exhibits the smallest losses. The results also indicate that the losses of the knitted antennas improve as the knitted patches became denser.

The presentation will include measured radiation patterns, efficiency results and an in-depth analysis of the strengths and weaknesses of this manufacturing technique.

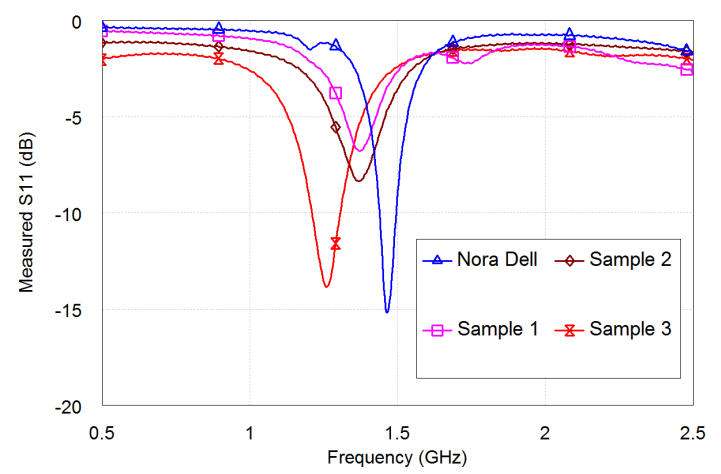

